\section{microRNA and lineage choice}

The microRNA miR-34 is transcriptionally induced by the tumor suppressor p53 and represses genes involved in cell-cycle progression, apoptosis and cell growth. In Immunity, Baltimore and colleagues show that constitutive expression of miR-34a causes a partial block in B cell development at the transition from pro-B cells to pre-B cells. Loss-of-function and rescue experiments indicate that Foxp1 and not Bcl-2, another previously described miR34a target, is the key mediator of the effects of miR-34a in this developmental context. Foxp1 is required at early stages of B cell development, during which it activates transcription factors such as E2A and Pax5 and binds to the enhancers of the recombinaseactivating genes Rag1 and Rag2. These results indicate that by inducing small changes in gene expression and controlling the balance between critical lineage-specification transcription factors, microRNAs can have an important role in cell fate. IV Immunity 33, 48-59 (2010)

\section{Competition breeds virulence}

Evolutionarily speaking, bacterial virulence is a puzzling phenomenon, especially when it does not correlate with any obvious growth or transmission advantage or results in damage and death to the host. In Current Biology, Weiser and colleagues offer a possible resolution to this paradox by taking into account an underappreciated parameter: in-host competition among colonizing bacteria. Using a modeling and experimental infection approach with the common opportunistic pathogens Haemophilus influenzae and Streptococcus pneumoniae, the authors find that the former can outcompete the latter by co-opting the function of neutrophils and complement. Specifically, components of $H$. influenzae (but not those of S. pneumoniae) stimulate complement-dependent phagocytic killing of $S$. pneumoniae, which then retaliates by producing a polysaccharide capsule able to resist opsonophagocytosis. As long as the cost of producing the capsule is not excessive, S. pneumoniae competes effectively, but the extent of encapsulation is the key component underlying bacterial virulence. This study emphasizes the fact that opportunistic infections should not be considered in isolation and that interbacterial conflict can drive pathogenicity.

ZTF

Curr. Biol. 20, 1222-1226 (2010)

\section{Controlling T cell activation}

The transcription factor $\mathrm{Bcl}-11 \mathrm{~b}$ has been described as a critical regulator of $\mathrm{T}$ lineage commitment at the double-negative 2 stage of T cell development. In the Journal of Experimental Medicine, Avram and colleagues use conditional deletion in mature $T$ cells to show that it also controls antigen-specific population expansion and cytolytic potential of $\mathrm{CD} 8^{+} \mathrm{T}$ cells during an immune response. Expression of the CD8 coreceptor and the signaling mediator PLC- $\gamma \mathbf{1}$ is lower in Bcl-11b-deficient cells, which suggests that suboptimal TCR signaling might explain the proliferation defect. The lower numbers of effector cytotoxic lymphocytes generated in these mice also have altered production of perforin and granzyme $\mathrm{B}$. Bcl-11b does not control CD8 expression in double-positive thymocytes, which suggests stage-specific developmental control in mature T cells. J. Exp. Med. (26 July 2010) doi:10.1084/jem.20092136

\section{Low-life fish; high-class immunity}

Basal chordates such as the lamprey have B cell- and T cell-like lymphocytes that express diverse antigen-specific receptors based on leucine-rich repeat modules. In the Proceedings of the National Academy of Science, Mariuzza and colleagues have now determined the structure of VLRA, the lamprey equivalent of the T cell antigen receptor (TCR), conjugated to the nominal antigen hen egg lysozyme (HEL). Unlike recognition by mammalian $\alpha \beta$ TCRs, recognition of antigen by VLRA has no requirement for antigen processing. Instead, it can bind antigen directly, much like certain mammalian $\gamma \delta$ TCRs. Another notable property of the VLRAHEL complex is that it binds with extremely high affinity (in the nano- to picomolar range), which would be expected more from an affinity-maturated immunoglobulin G antibody than a TCR-like molecule. The VLRA-HEL complex therefore demonstrates some striking parallels to both mammalian $\gamma \delta$ TCRs and antibodies. It remains to be determined whether other VLRA subsets can recognize antigen in the context of an analog to the major histocompatibility complex.

Proc. Natl. Acad. Sci. USA 107, 13408-13413 (2010)

\section{Dual role for PTIP}

Immunoglobulin class-switch recombination (CSR) replaces the $\mu$-heavy chain with another isotype encoded by downstream constant-region exons, preserving antigen specificity but tailoring effector function. In Sciencexpress, Daniel et al. examine activated B cells lacking PTIP, a component of the methyltransferase complex MLL3-MLL4 that catalyzes di- and trimethylation of Lys4 on histone H3 (H3K4). After being stimulated with lipopolysaccharide, PTIP-deficient B cells show less H3K4 trimethylation (H3K4me3) in the $\mathrm{S}_{\gamma} 2 \mathrm{~b}, \mathrm{~S}_{\gamma} 3$ and $\mathrm{S}_{\gamma} 1$ regions of the immunoglobulin heavy-chain locus but have equivalent amounts of $\mathrm{H} 3 \mathrm{~K} 4 \mathrm{me} 3$ at $\mathrm{S}_{\mu}$. These changes correlate with less histone acetylation and binding of RNA polymerase II and the generation of fewer sterile transcripts at the downstream constant regions. As transcription seems to be required for targeting these loci for activation-induced cytidine deaminase-dependent recombination, CSR frequency is likewise lower in PTIP-deficient B cells. PTIPdeficient $B$ cells also have more genomic instability, which indicates that repair of double-stranded DNA breaks requires PTIP. Thus, PTIP acts at many steps in CSR.

Sciencexpress (29 July 2010) doi:10.1126/science. 1187942

\section{ATP regulates NK cells}

The cytolytic activity of human natural killer (NK) cells can be enhanced after exposure to the inflammatory chemokine CX3CL1 (fractalkine), which is expressed on damaged endothelial cells. In Blood, Gorini et al. show that ATP released by healthy endothelial cells blunts NK cell chemotaxis toward the chemokine CX3CL1 and inhibits enhancement of cytolytic activity. Treatment of endothelial cells with apyrase enhances their susceptibility to being killed by NK cells, which suggests that endogenous ATP protects these cells. ATP as well as NAD ${ }^{+}$specifically activate the $\mathrm{P}_{2} \mathrm{Y}_{11}$ nucleotide receptor expressed on NK cells. This interaction leads to an increase in cytosolic intracellular cAMP and an influx of extracellular $\mathrm{Ca}^{2+}$. In turn, the increase in intracellular cAMP acts to inhibit downstream signaling via the CX3CL1 receptor CX3CR1 in an unspecified way. Notably, ATP does not alter NK cell chemotaxis toward another chemokine, CXCL12, nor is NK cell survival affected. How this specificity in chemotactic responses is attained remains a topic for future work.

$L A D$

Blood (28 July 2010) doi:10.1182/blood-2009-12-260828 